\title{
Predictive Geometric Models for Heat-Insulation Properties of Semi-Finished Fur and Down Products
}

\author{
Victor Yurkov ${ }^{1,2}$, Elena Dolgova ${ }^{1}$ and Margarita Chizhik ${ }^{1}$ \\ ${ }^{1}$ Omsk State Technical University, 11 Mira Prospekt, Omsk, 644011, Russia \\ ${ }^{2}$ Omsk State Pedagogical University, 14 Tuhachevsky Embankment, Omsk, 644099, Russia
}

\begin{abstract}
This paper is devoted to geometric simulation of heat-insulation properties of fur and down products which are considered as multi-parameter and multi-component systems. We consider predictive models of heat resistance depended on physical characteristics of fur and pelt. There is a problem of construction co-ordinate geometric models on condition that the set of experimental data is limited. We solve the problem as a problem for static multi-component systems. The model is considered as a piecewise constant function in the space of input and output parameters. The paper proposes an algorithm of construction the clusters on the set of given experimental points. Moreover, we construct multidimensional convex covering on the set of the points. The covering is based on its two-dimensional projections. Results of the investigations allow us to substantiate producer's choice of fur and down semi-finished products and its composition for manufacturing the product of special purpose. The method suggested in the paper may be one of geometric modulus of the software HYPER-DESCENT which has been developed formerly. Our geometric models together with software HYPERDESCENT may be applied for simulation and prediction the properties of another multiparametrical systems or technological processes of light industry.
\end{abstract}

\section{Keywords}

Multicomponent system, fur semi-finished product, heat-protective properties, geometric modeling, clustering.

\section{Introduction}

At present there are many problems connecting with technical control and prediction various properties of fur and down products. Real fur and fur clothes have high heat-insulation properties. All properties are conditioned by slight air permeability of leather and high heat resistance of hair covering that causes an air layer. All heat-insulation properties of fur semi-finished products depend on the length and density of the hair and also these properties depend on thickness and density of the leather. That leads not only to various versions of constructive models but to various combinations of materials in the same heat-insulation multi-layer package. At present all classifications of heat-insulation properties for fur and down semi-finished products are relative ones because heat resistance depends not only on properties of the pelt but it depends on the breed, quality of the pelt, finishing operations and so on. Therefore, elaboration of new methods and design of predictive geometric models for fur and down products are actual problems at present.

As for geometric aspect of the problem it is necessary to take into account the following circumstances. Firstly, there is a problem of multi-factorial nature of objects. A great number of factors are inaccessible and indeterminate. Hence, we have a problem of data analyses. Secondly, one can be faced with different experimental output results even if all input parameters are the same ones. It means existence of some unknown factors. Thirdly, some of input parameters may be fuzzy. And at last if we want to find a proper predictive model as a monoidal hyper-surface in the space of input and output

GraphiCon 2021: 31st International Conference on Computer Graphics and Vision, September 27-30, 2021, Nizhny Novgorod, Russia EMAIL: viktor_yurkov@mail.ru (V. Yurkov); dolgova13@rambler.ru (E. Dolgova); margarita-chizhik@ rambler.ru (M. Chizhik) ORCID: 0000-0003-2667-8103 (V. Yurkov); 0000-0003-3174-7142 (E. Dolgova); 0000-0003-0797-875X (M. Chizhik)

(c) (i) 2021 Copyright for this paper by its authors.

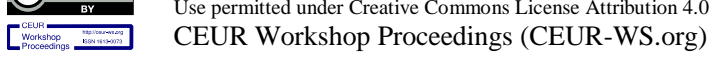


parameters we must have a great number of experimental samples given by an appointed plan. But it is impossible because it is impossible to rear the animals with beforehand given properties of their fur and pelt.

Limited number of experimental data requires realization of original modeling approachs. We analyze all data and appraise possibility of separation the data into clusters. Then we simulate properties of the clusters by means of piecewise constant functions. As we accumulate enough number of data we may simulate some cluster properties by polynomials. Finally, we may find the same piecewise model for input parameters area as a single whole $[1,2]$.

\section{Object and aim of the researching}

The aim of this paper is to give a description on analytic representation of heat-insulation properties for fur and down products.

As an object of investigation we were given several samples of rabbit pelt only. Physical parameters of the pelt are in Tabl. 1.

Table 1

Mass and geometric characteristics of rabbit pelt

\begin{tabular}{cccc}
\hline $\begin{array}{c}\text { Thickness of leather, } \\
\mathrm{mm}\end{array}$ & $\begin{array}{c}\text { Length of the fue } \\
\text { hair, } \mathrm{mm}\end{array}$ & $\begin{array}{c}\text { Density of the leather, } \\
\mathrm{g} / \mathrm{cm}^{3}\end{array}$ & $\begin{array}{c}\text { Density of the hair cover, } \\
\mathrm{g} / \mathrm{cm}^{3}\end{array}$ \\
\hline $0.95 \pm 0.1$ & $6 \pm 0.5$ & $1.50 \pm 0.2$ & $0.056 \pm 0.005$ \\
$0.43 \pm 0.05$ & $9 \pm 1.0$ & $0.69 \pm 0.07$ & $0.048 \pm 0.005$ \\
$0.53 \pm 0.05$ & $25 \pm 2.5$ & $0.63 \pm 0.07$ & $0.025 \pm 0.005$ \\
$0.59 \pm 0.05$ & $15 \pm 1.5$ & $0.66 \pm 0.07$ & $0.033 \pm 0.005$ \\
$0.51 \pm 0.05$ & $10 \pm 1.0$ & $0.48 \pm 0.05$ & $0.040 \pm 0.005$ \\
$0.75 \pm 0.07$ & $9 \pm 1.0$ & $0.47 \pm 0.05$ & $0.033 \pm 0.005$ \\
$0.51 \pm 0.05$ & $5 \pm 0.5$ & $0.61 \pm 0.07$ & $0.027 \pm 0.005$ \\
\hline
\end{tabular}

\section{General theoretical considerations}

In this paper we consider one static mathematical model of multi-component system [3, 4]. We are limited both in the number of input parameter realizations and in corresponding realizations of output parameters. Limited sets of input and output parameters are considered as initial data of modeling. Let $\boldsymbol{X}$ be a set of input parameters and let $\boldsymbol{Y}$ be a set of output parameters of the system. Our purpose is to identify a nonlinear correspondence $\boldsymbol{Y}=F(\boldsymbol{X}, \boldsymbol{A})$. Specifically, we have: $\operatorname{dim} \boldsymbol{X}=n, \operatorname{dim} \boldsymbol{Y}=1$.

Special feature of the problem is that all realizations of parameters are random. The structure of the model may be chosen subjectively or it may be identified objectively. If the structure has $m$ numerical parameters we need $m$ random realizations at the minimum. Moreover, preliminary structure analyses of geometric data may be at variance with a formal mathematical model. For example, structural contradiction of data and formal model is shown in Figure 1. To eliminate the contradiction one can use a piecewise constant model which is shown by dash lines. Using a more complicated model we take a risk to have an absurd result.

If the number of parameters for linear model exceeds the number of given realizations we have some indetermination of the model. And at last geometric structure of data may be unfit for extrapolation. That takes place if values of input parameters have comparatively small differences but corresponding values of output parameters have a great differences.

We begin by consideration the space $\boldsymbol{X} \times \boldsymbol{Y}, \operatorname{dim}(\boldsymbol{X} \times \boldsymbol{Y})=n+1$, having a rectangular $n$-dimensional area $X: a_{i} \leq x_{i} \leq b_{i} . i=1, \ldots, n$, and a section $Y: y_{\min } \leq y \leq y_{\max }$. Probable locations of the area $X$ and the section $Y$ are determined by a priory information and physical meaning of data. In $X$ we have finite set $\boldsymbol{P}$ of points $P_{i}, i=1, \ldots, p$. Each point $P_{i} \in \boldsymbol{P}$ is considered as a centre of some $n$-dimensional rectangular 
subset $\left(x_{1} \pm a_{1}, x_{2} \pm a_{2}, \ldots, x_{n} \pm a_{n}\right)$. The number of points $P_{i}$ may be lacking for finding a polynomial model and all locations of the points may be chaotic in $X$. All given points may generate some compact groups or they may be located more or less evenly. Being limited by all these conditions we want to find an analytic model $y=f\left(x_{1}, \ldots, x_{n}\right)$ of the system and also we need possibility of extrapolation the values of $y$ onto the points which will be given out of the set $\boldsymbol{P}$.

In order to solve the problem we suggest several stages. At first stage we construct a multidimensional convex covering of the finite set $\boldsymbol{P}$ [5]. First principals of covering are illustrated by Figure 2.
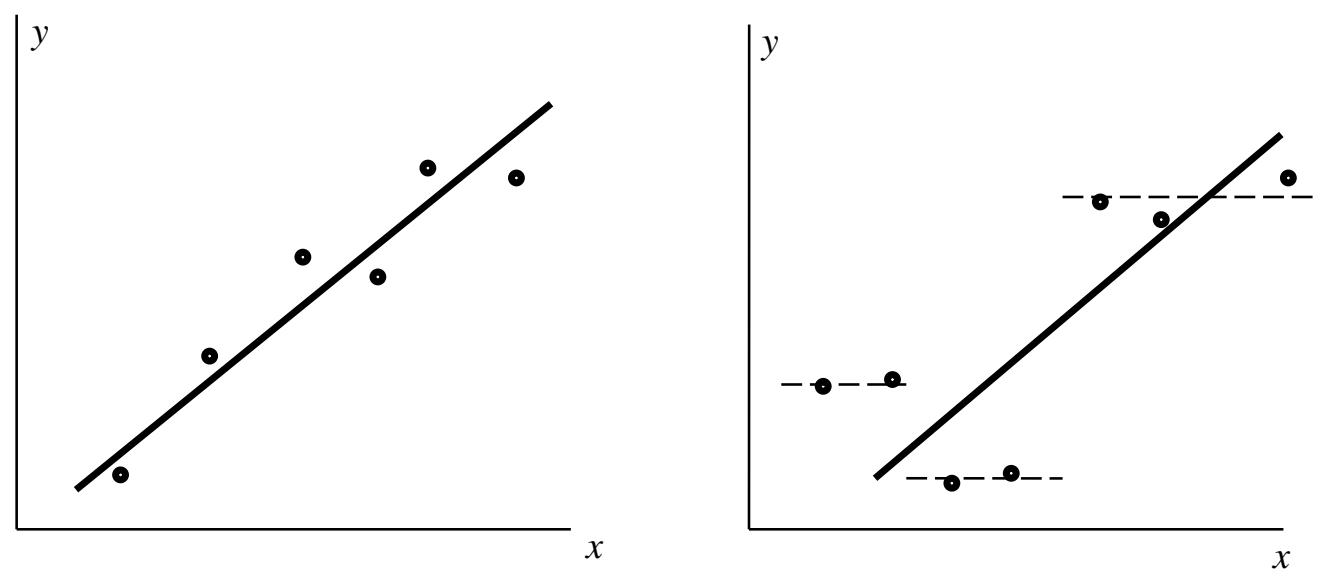

Figure 1: The model is in accordance with the data (on left side) and the model is not in accordance with the data (on right side)

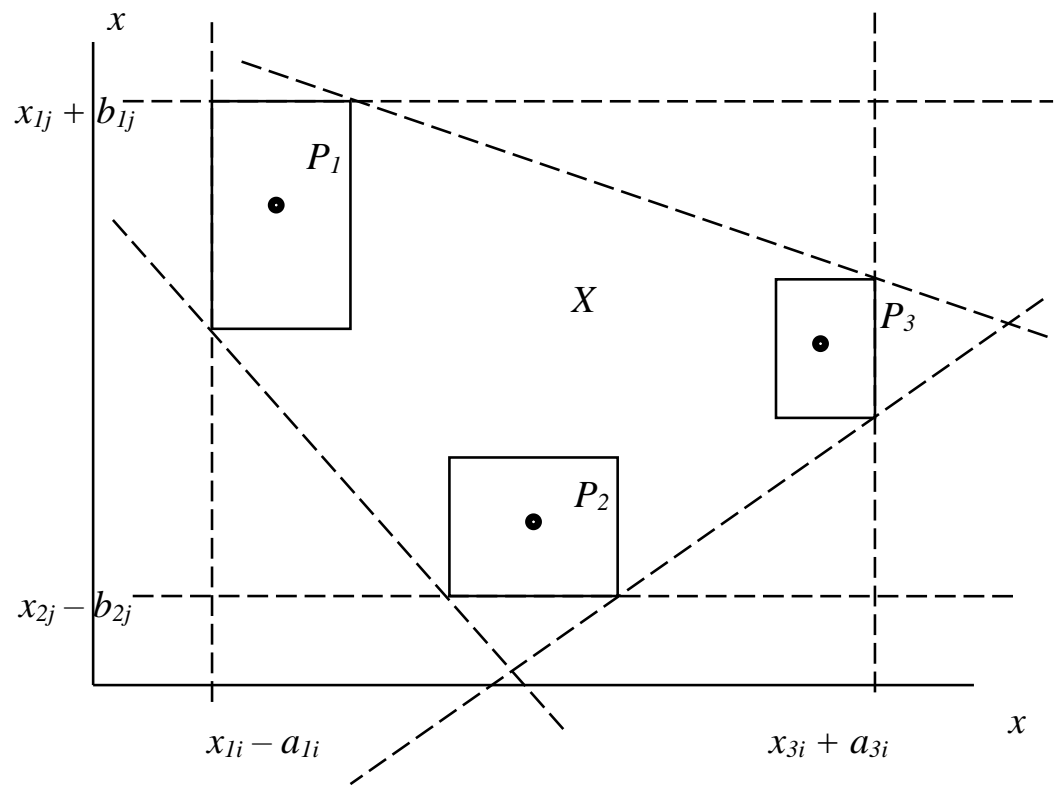

Figure 2: Two-dimensional convex covering $X_{i j}$ of the set $P_{1}, P_{2}, P_{3}$

We have:

$$
\begin{gathered}
P_{1}\left(x_{1 i} \pm a_{1 i}, x_{1 j} \pm b_{1 j}\right), P_{2}\left(x_{2 i} \pm a_{2 i}, x_{2 j} \pm b_{2 j}\right), P_{3}\left(x_{3 i} \pm a_{3 i}, x_{3 j} \pm b_{3 j}\right), \\
X_{i j}=\left\{x_{1 i}-a_{1 i} \leq x_{i} \leq x_{3 i}+a_{3 i}\right\} \cap\left\{x_{2 j}-b_{2 j} \leq x_{j} \leq x_{1 j}+b_{1 j}\right\} \cap \\
\cap\left\{x_{j} \geq f\left(x_{1 i}+a_{1 i}, x_{1 j}+b_{1 j}, x_{3 i}+a_{3 i}, x_{3 j}+b_{3 j}\right)\right\} \cap \\
\cap\left\{x_{i} \geq f\left(x_{1 i}-a_{l i}, x_{l j}-b_{l j}, x_{2 i}-a_{2 i}, x_{2 j}-b_{2 j}\right)\right\} \cap\left\{x_{i} \leq f\left(x_{2 i}+a_{2 i}, x_{2 j}-b_{2 j}, x_{3 i}+a_{3 i}, x_{3 j}-b_{3 j}\right)\right\} .
\end{gathered}
$$


Here $X_{i j}$ is a projection of the covering onto the plane $O x_{i} x_{j}$. All combinations of two-dimensional projections of the set $\boldsymbol{P}$ and all their convex coverings are constructed by the conditions $i=1, \ldots, n-$ $1 ; j=2, \ldots, n ; j>i$. As a result we have $n$-dimensional convex covering $X$ of the set $\boldsymbol{P}: X=\cap X_{i j}$.

The second stage includes some data clustering algorithms [6,7]. All algorithms are based on supposition that geometric nearness of points $P_{i}$ and $P_{j}, i \neq j$, means a physical closeness of its originals. Complete number of clusters is unknown beforehand. It may be in interval from 1 up to $p$. Nearness of points is considered as a distance $d\left(P_{i}, P_{j}\right)$ in Euclidean or Hamming metrics.

As for the space $\boldsymbol{Y}$ it may be divided into strata $Y_{k}=\left\{y_{k}: y_{k}{ }^{m i n} \leq y_{k} \leq y_{k}{ }^{\max }\right\}, 1 \leq i \leq p$. In general case we have $\cup Y_{k} \neq \boldsymbol{Y}$ and a number of strata may be in interval from 1 up to $p$. Points $P_{i} \in \boldsymbol{P}$ are united at one cluster if $y_{k}^{\min } \leq y_{k}\left(P_{i}\right) \leq y_{k}^{\max }$. After that we verify connexity of the clusters. Any of clusters is considered as a connected one if all its points $P_{i}$ are in the same stratum. If it is not so the cluster is considered as a disconnected one and it is divided in two or more parts that are new clusters.

At the third stage we divide the area $X$ into sub-areas having closeness of their physical parameters. Since the number and location of all clusters are known it is necessary to find geometric place of points which distance to given cluster is less then distances to all another clusters. In other words we have to construct some similarity of Voronoi diagram of order $k$ in multidimensional space. That problem is connected with considerable algorithmic difficulties and that is why we prefer more simple approximating algorithms. Its principle is shown in Figure 3.

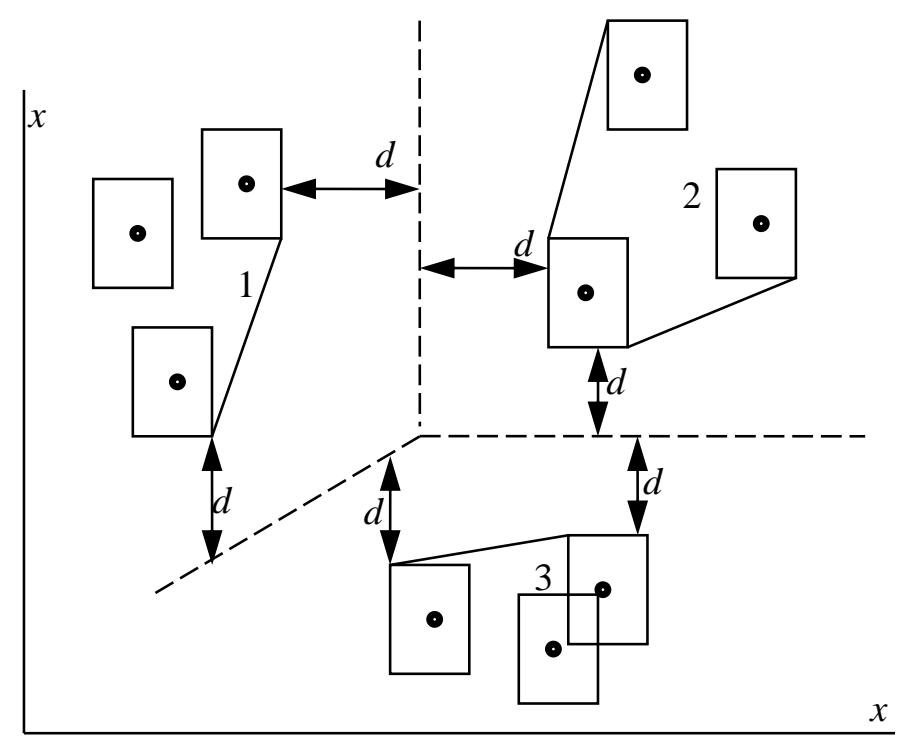

Figure 3: Construction of separating hyper-planes for the clusters 1, 2 and 3 in Hamming metric

We construct convex envelopes for all clusters. Each convex envelope is a closed broken line, if $n=$ 2 , or it is a multidimensional closed polyhedron, if $n>2$. Separation of one envelope from another is realized by hyper-planes. The set of separating hyper-planes consists of hyper-planes which are parallel to coordinate $(n-i)$-dimensional spaces, where $i=1, \ldots, n-1$. For example, all separating hyperplanes shown in Fig. 3 are hyper-planes perpendicular to coordinate plane $O x_{i} x_{j}$. The hyper-planes which separate two neighbouring clusters are construct as hyper-planes equidistant to these clusters.

Our fourth stage includes the construction of piecewise constant data approximation. Constant function

$$
y_{k}=\Sigma y_{i} / p_{k}, P_{i}\left(x_{1 i}, \ldots, x_{n i}, y_{i}\right) \subset X_{k} \times \boldsymbol{Y}_{k}, i=1, \ldots, p_{k}
$$

is constructed in each stratum. To illustrate constructive principle some conventional monotonous piecewise constant model which approximate the data for three connected clusters and three separated strata $\mathrm{Y}_{1}, \mathrm{Y}_{2}, \mathrm{Y}_{3}$ is shown in Figure 4. 


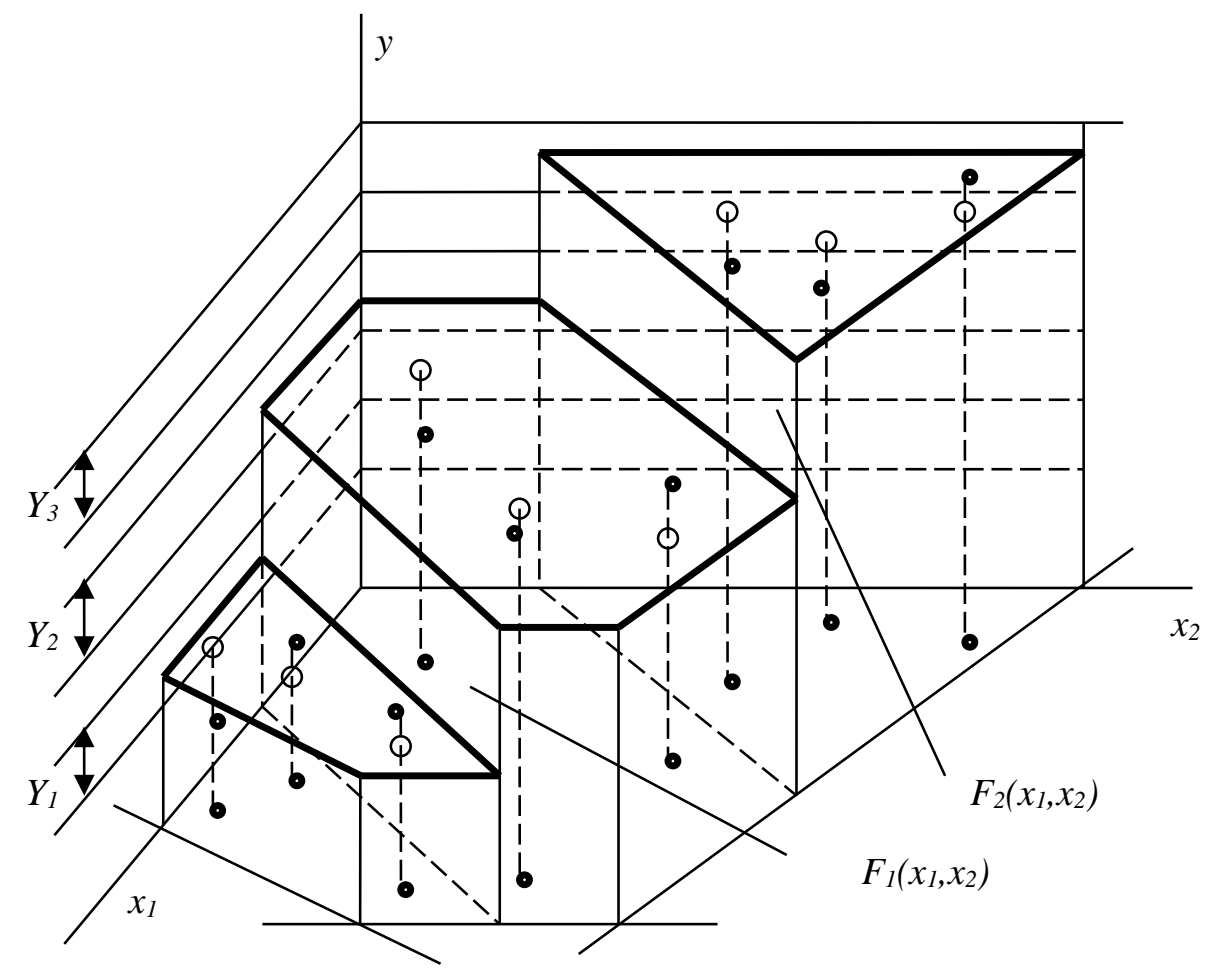

Figure 4: Piecewise constant approximation of three clusters and three strata

\section{Method of research, fabrics and equipments}

Total heat-insulation was determined by original method which has been developed and patented beforehand. The gist of the method was the following (see Figure 5). A heat accumulator was placed into the packet made of fur and down semi-finished materials. The temperature was varied purposefully. The moment of heat accumulator cooling was recorded. Calculation of total heat-insulation was computed by formula $R=(S \cdot t) /(C \cdot m)$, where $S$ is an area of the sample, $t$ is total time of cooling, $c$ is a heat capacity of the sample and $m$ is a mass of accumulator. The results are shown in Tabl. 2 .

Table 2

The values of total heat resistance

\begin{tabular}{cc}
\hline Time of cooling, $\mathrm{s}$ & Heat resistance, $\mathrm{m}^{2} \mathrm{~K} / \mathrm{Wt}$ \\
\hline 5160 & 0.681 \\
4680 & 0.617 \\
4800 & 0.634 \\
4740 & 0.625 \\
4560 & 0.602 \\
4320 & 0.570 \\
3660 & 0.483 \\
\hline
\end{tabular}

Thickness of the leather was measured by thickness gauge and the length of the hair was measured by a special measuring bar.

Original device for heat-insulation measuring consists of heat accumulator which has a special gel and hermetic container. The latter is a hollow cylinder and the sample is wound round it. The sample consists of one or several pelt strips. The length of the pelt strips is varied from 186 up to $378 \mathrm{~mm}$. The strip is from 20 up to $140 \mathrm{~mm}$ wide. 


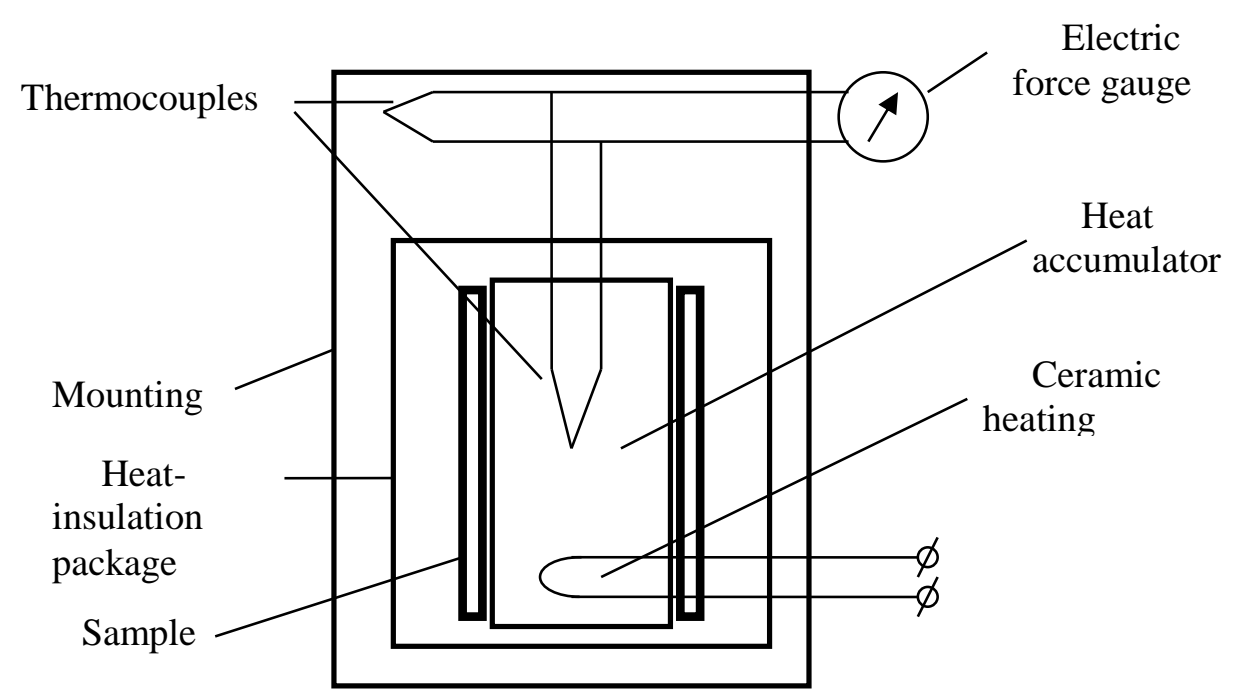

Figure 5: The device for total heat resistance determination

\section{Predictive model for heat resistance}

Let $x$ be a thickness of the leather, $y$ be a length of the hair, $z$ be a density of leather texture, $v$ be a closeness of hair covering and $t$ be a heat resistance. After experimental researches of all given fur and down samples we have the following mathematical model of heat resistance. Four-dimensional covering $X=X_{x y} \cap X_{x z} \cap X_{x v} \cap X_{y z} \cap X_{y v} \cap X_{z v}$ of the set $\boldsymbol{P}$ is described by the following inequalities

$X_{x y}: 5 \leq y \leq 25 ; 150 x-50 \leq y \leq-50 x+55 ; 0.4 \leq x \leq 1$;

$X_{x z}: 0.4 \leq x \leq 1 ; 0.4 \leq z \leq 1.5 ; \mathrm{z} \leq 1.6 x+0.06$;

$X_{x v}: 0.4 \leq x \leq 1 ; 0.02 \leq v \leq 0.04 ;(4 / 30) x-2.2 / 30 \leq v \leq(1 / 60) x+2.6 / 60$;

$X_{y z}: 5 \leq y \leq 25 ; 0.4 \leq z \leq 1.5 ; z \leq-(0.8 / 19) y+28.98 / 19$;

$X_{y v}: 5 \leq y \leq 25 ; 0.02 \leq v \leq-(0.03 / 20) y+1.35 / 20$

$X_{z v}: 0.4 \leq z \leq 1.5 ; 0.02 \leq v ;(1 / 30) z \leq v \leq(1 / 110) z+5.1 / 110$.

Formal clustering algorithm gives us the following results $C L_{1}=\left\{P_{1}\right\}, C L_{2}=\left\{P_{2}, P_{4}, P_{5}, P_{6}\right\}, C L_{3}$ $=\left\{P_{3}\right\}, C L_{4}=\left\{P_{7}\right\}$.

The corresponding coverings are the following

$X_{1}: x>0.7 ; y<7 ; z>1 ; v>0.04 ; x / 1.3+v / 0.09>1$;

$X_{2}: 7<y<20 ; x / 1.3+v / 0.09<1 ; x / 0.75+v / 0.057>1 ; z<1 ; v>0.03$;

$X_{3}: y>20 ; z<1 ; x / 0.75+v / 0.057<1 ; v<0.03$

$X_{4}: x<0.7 ; y<7 ; z<1 ; x / 0.75+v / 0.057<1 ; v<0.03$.

Heat resistances are

$t\left(X_{1}\right)=0.681 \pm 0.07 ; t\left(X_{2}\right)=0.6 \pm 0.06 ; t\left(X_{3}\right)=0.634 \pm 0.06 ; t\left(X_{4}\right)=0.483 \pm 0.05$.

\section{Conclusions}

We have obtained the piecewise constant analytical model of heat-insulation for fur and down semifinished products. Heat resistance of the products depends upon superficial physical characteristics of fur and pelt. The physical characteristics are partly determined by thickness and density of the leather, length and closeness of the hair covering.

Using the investigations we can motivate our selection of components and the structure of fur and down fabrics which are used for manufacturing the special products. Using the analytical descriptions of heat-insulation we can forecast its values before manufacturing $[8,9]$. The method of predictive geometric models may be one of the modulus of software HYPER-DESCENT and it may allow us to solve the predictive problems [10].

Throughout the paper we have required the piecewise regularity of the model. Taking that into account we may note the partial solution of the problem of searching optimal correlation between 
structure properties of fur and down pelt and heat-insulation properties of the products. We think that general solution of the problem needs further investigation. Another direction in which the theory could be generalized is to investigate the problem of designing multi-component products having beforehand given properties.

\section{References}

[1] H. Park, K.Kim, An adaptive method for smooth surface approximation to scattered 3D points, Computer Aided Design 28.12 (1996) 995 - 1005.

[2] M. A. Chizhik, V. Yu. Yurkov and E. Yu. Dolgova, Geometric models of multi-parametric technological processes for estimation some inverse control problems, Journal of Phisics: Conference Series. XIV International Scientific and Technical Conference "Applied Mechanics and Systems Dymamics" (2021) 012043.

[3] R. Z. et al Farahani, Covering problems in facility location: A review, Computers and Industrial Engineering 62 (2012) 368-407.

[4] Y. Buhaienko, L. S. Globa, A. Liashenko and M. Grebinechenko, Analyses of clustering algorithms for use in the universal data processing system, Opened semantic technologies of intellectual system design 4 (2020) $101-104$.

[5] S. K. Dulin, I. N. Rosenberg, V. I. Umansky, Clustering methods in investigation of geo-data, Systems and methods of informatics 19.2 (2009) $86-113$.

[6] H. Stachel, H. Abdelmoez, Voronoi Diagrams and offsets, an Algorithm based on Descriptive Geometry, Proceeding of Compugraphics (1992) $159-166$.

[7] E. Remy, E. Thiel, Axis for chamfer distances: computing look-up tables and neighbourhoods in 2D or 3D, Pattern Recognition Letters 23.6 (2002) 649 - 661.

[8] M. A. Chizhik, E. Yu. Dolgova and T. M. Ivantsova, The method of heat-insulation properties determination of materials and packages, Patent No. 2527314 Russia. Filed December 19, 2012. Issued August 27, 2014.

[9] C.-Y. Hu, T. Maekava, E. C. Sherbrooke and N. M. Patrikalakis, Approximation of measured data with interval B-splines, Computer Aided Design 29 (1997) 791 - 799.

[10] M. A. Chizhik, M. N. Moskovtsev, D. P. Monastyrenko and D. V. Dorkin, State registration of software Hyper-descent, No. 2014610165. Filed July 19, 2013. Issued January 09, 2014. 